\title{
Study of high muon multiplicity cosmic-ray events with ALICE at the CERN Large Hadron Collider
}

\section{Rodríguez Cahuantzi* for the ALICE Collaboration ${ }^{\dagger}$}

Faculty of Physics and Mathematics, Autonomous University of Puebla, México

E-mail: mrodriguez@fcfm.buap.mx, mrodrigu@mail.cern.ch

\begin{abstract}
ALICE is one of four large experiments at the CERN Large Hadron Collider. Located 52 meters underground with 28 meters of overburden rock, it has also been used to detect atmospheric muons produced by cosmic-ray interactions in the upper atmosphere. We present the muon multiplicity distribution of these cosmic-ray events and their comparison with Monte Carlo simulation. This analysis exploits the large size and excellent tracking capability of the ALICE Time Projection Chamber. A special emphasis is given to the study of high multiplicity events containing more than 100 reconstructed muons and corresponding to a muon areal density larger than $5.9 \mathrm{~m}^{-2}$. The measured rate of these events shows that they stem from primary cosmic-rays with energies above $10^{16} \mathrm{eV}$. The frequency of these events can be successfully described by assuming a heavy mass composition of primary cosmic-rays in this energy range and using the most recent hadronic interaction models to simulate the development of the resulting air showers.
\end{abstract}

The 34th International Cosmic Ray Conference,

30 July- 6 August, 2015

The Hague, The Netherlands

\footnotetext{
*Speaker.

†Co-authors: B. Alessandro (INFN, sez. Torino, Italy), A. Fernández Téllez (Autonomous University of Puebla, México), K. Shtejer Díaz (CEADEN, Cuba) and E. González Hernández (Autonomous University of Puebla, México)
} 


\section{Introduction}

The study of atmospheric muons using collider experiments started during the era of the Large Electron Positron collider (LEP) by ALEPH and DELPHI. The main result reported by the LEP experiments in the area of cosmic-ray physics was the observation of muon-bundle events that could not be described within the existing hadronic interaction models. Even with the combination of extreme assumptions of highest-measured flux value and pure-iron spectrum, the Monte Carlo models at that time failed to describe the abundance of events with high multiplicity of muons $[1,2]$.

A development of this program of study is possible at the LHC, where experiments operate for many years, with the possibility of recording a large amount of cosmic-ray data. In this context, although the main purpose of ALICE is the study of beam-induced collisions, the experiment can also operate to detect muons produced by cosmic-ray interactions with the atmosphere [3]. Therefore, ALICE began a program to record cosmic-ray data, collecting about 31 effective days of data between 2010 and 2013 (around 22.6 million events recorded with at least one reconstructed muon), during pauses in LHC operation. Additionally, in 2012 a special trigger configuration allowed a test of the detection of cosmic events during proton-proton collisions runs.

The muons collected in ALICE are created in extensive air showers (EAS) following the interaction of cosmic-ray primaries with the nuclei of the atmosphere. Accordingly with the Monte Carlo study conducted in this work, the EAS observed by ALICE with muon multiplicity larger than four, are due to primaries with energy $E>10^{14} \mathrm{eV}$.

The detection of EAS has been done by many large-area apparatus located at the surface [4-7], while deep-underground detectors [8-10] have studied the high energy muons of the EAS. The big advantages of the surface apparatus are their large size and the possibility of measuring different particles, such as electrons, muons and hadrons created in the EAS. Since the detectors involved in collider experiments are tiny compared with the large area surface arrays, the approach and the studies have to be different.

In this work we have used the ALICE Time Projection Chamber (TPC) [11] to study multimuon events (events with more than four reconstructed muons). The Monte Carlo simulations were based on QGSJET $[12,13]$, a hadronic interaction model commonly used in EAS simulations.

Note: A publication on this topic was released by the ALICE Collaboration two days before the scheduled talk given at ICRC 2015. To avoid overlapping between the content of this paper and the general ALICE publication, only preliminary results (which do not change the message of the present study) are shown in this manuscript. To consult the results as presented at ICRC 2015, follow the reference of the ALICE publication [18].

\section{Experimental setup}

ALICE is located at Point 2 of the LHC accelerator tunnel, in a cavern placed $52 \mathrm{~m}$ underground and with $28 \mathrm{~m}$ overburden of rock, at $450 \mathrm{~m}$ above the sea level. The rock absorbs all of the electromagnetic and hadronic components of an EAS, while near vertical muons with a surface energy greater than $16 \mathrm{GeV}$ can reach the detectors [14]. ALICE is a typical collider experiment, with a solenoid magnet housing the central barrel detector, and a forward muon arm, consisting of 
absorbers, a large dipole magnet, and fourteen planes of tracking and triggering chambers located outside the ALICE magnet. A complete description of the apparatus is given in [3].

The ALICE TPC was used to reconstruct the trajectory of cosmic-ray muons passing through the active volume of the detector. For the purpose of detecting cosmic-ray muons, the effective area of the detector considering its horizontal cylindrical geometry is approximately $17 \mathrm{~m}^{2}$.

Specific triggers have been implemented to detect atmospheric muons crossing the central barrel of the ALICE apparatus (see Fig.1). To trigger and measure cosmic-ray events, three detectors were used: ACORDE (Alice COsmic Ray DEtector), TOF (Time of Flight) and SPD (Silicon Pixel Detector).

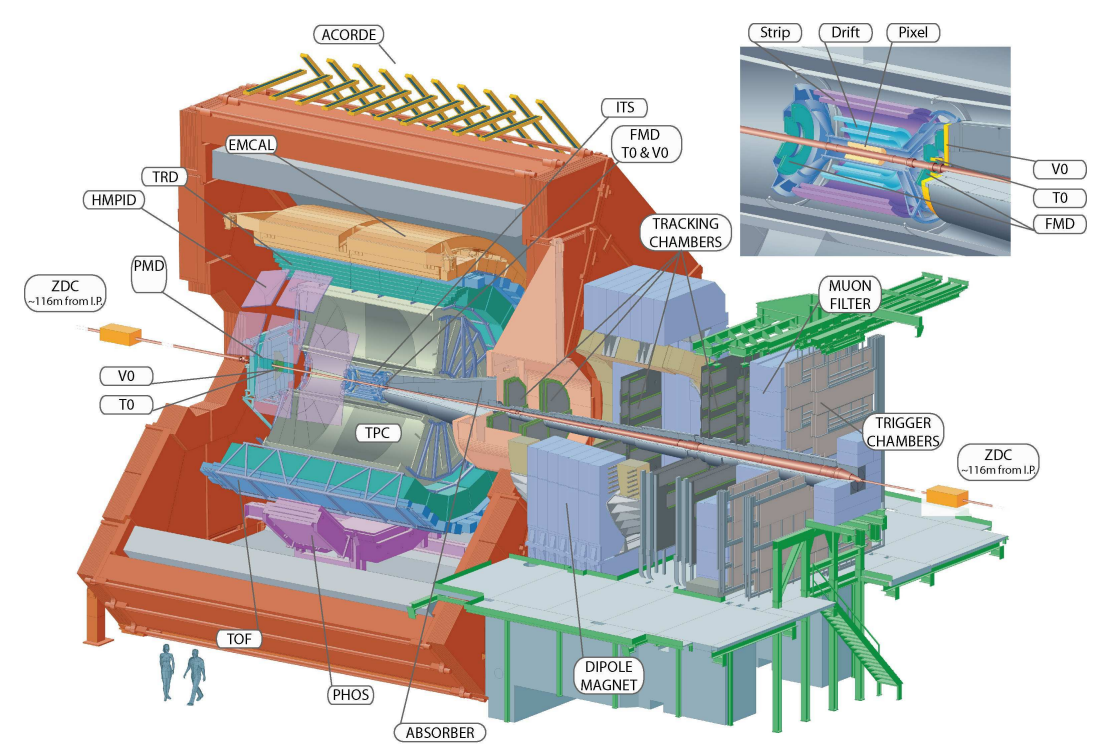

Figure 1: The ALICE detector at P2-LHC. ACORDE, TOF, TPC and SPD are used to trigger and measure cosmic-ray events.

ACORDE consists of an array of 60 scintillator modules located on the three top octants of the ALICE magnet. Each module is composed of two superimposed plastic scintillator paddles with an effective detection area of $0.37 \mathrm{~m}^{2}$ (see Fig. 2). The trigger is given by the coincidence of the signals in $n$ different modules ( $n$-fold coincidence) in a 100 ns time window. For this work, the trigger of ACORDE was configured with $n=4$.

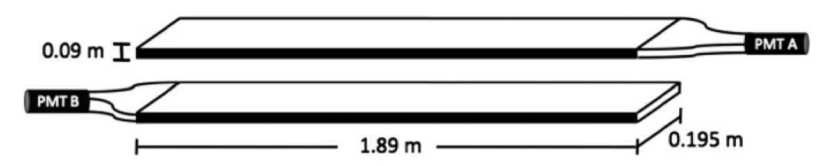

Figure 2: Schematic view of one ACORDE module. The light produced in the scintillator plastic is driven by two optical guides to one PMT per paddle. The information of the pulse is sent to the FEE (front end electronics) to generate the ACORDE cosmic-ray trigger.

The SPD is part of the Inner Tracking System located inside the inner field cage of the TPC. It is composed of two layers of silicon pixel modules located at a distance of $39 \mathrm{~mm}$ and $76 \mathrm{~mm}$ from the LHC beam axis, respectively. The layers have an active length of $28.3 \mathrm{~cm}$, centered upon 
the nominal interaction point of the LHC beams. The SPD was incorporated into the trigger by requiring a coincidence between signals in the top and bottom halves of the outermost layer.

TOF detector is a cylindrical MRPC (Multi-gap Resistive Plate Chamber) array, completely surrounding the TPC. The trigger requires a signal in a read-out channel (a pad) in the upper part of the TOF and another in a pad in the opposite lower part forming a back-to-back coincidence with respect to the central axis of the detector.

A logical OR among the trigger signals of ACORDE, TOF and SPD was configured to generate the cosmic-ray trigger of ALICE. Most events were classified as either single muon events or multimuon events, with a small percentage of "interaction" events where very energetic muons have interacted with the iron yoke of the magnet producing a shower of particles that pass through the TPC.

\section{Reconstruction of atmospheric muons}

The atmospheric muons that cross the ALICE central barrel are reconstructed as two tracks (see Fig. 3). In order to quantify the muon multiplicity per event, a dedicated algorithm was developed to match both reconstructed tracks and to obtain the full length of the muon track crossing the TPC. We refer to these tracks as up and down tracks. However, the tracking algorithm has not been optimized for very inclined (quasi horizontal) tracks. Therefore, to avoid reconstruction inaccuracies associated with the most inclined showers, we restricted the zenith angle of all events to the range $0^{\circ}<\theta<50^{\circ}$.

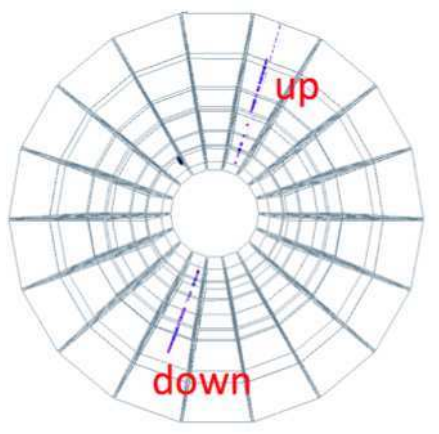

Figure 3: Track reconstruction of one single atmospheric muon with the TPC of ALICE.

Each TPC track can be reconstructed with up to 159 clusters (space points). In this analysis, we required tracks with at least 50 clusters in the TPC and a momentum larger than $0.5 \mathrm{GeV} / \mathrm{c}$. Since the atmospheric muons coming from the same EAS event arrive almost parallel at ground level, all the analyzed tracks per event should be parallel between them.

Finally, each up-track is matched to the closest down-track when the distance in the transverse plane (XZ) between them is less than $3 \mathrm{~cm}$. A muon reconstructed with two tracks is called "matched muon". If the matching condition is not fulfilled the track is anyway accepted as a muon and called "single-track muon". Most of the single-track muons originate from particles crossing the TPC close to its edge, where part of the muon trajectory may fall outside the detector. 


\section{Multiplicity distribution of atmospheric muons (MMD)}

The main topic related to cosmic-ray physics investigated by ALICE is the study of the muonmultiplicity distribution and in particular of events with high density of muons.

The atmospheric-muon multiplicity (MMD) distribution from the whole sample of our data set is shown in Fig. 4.

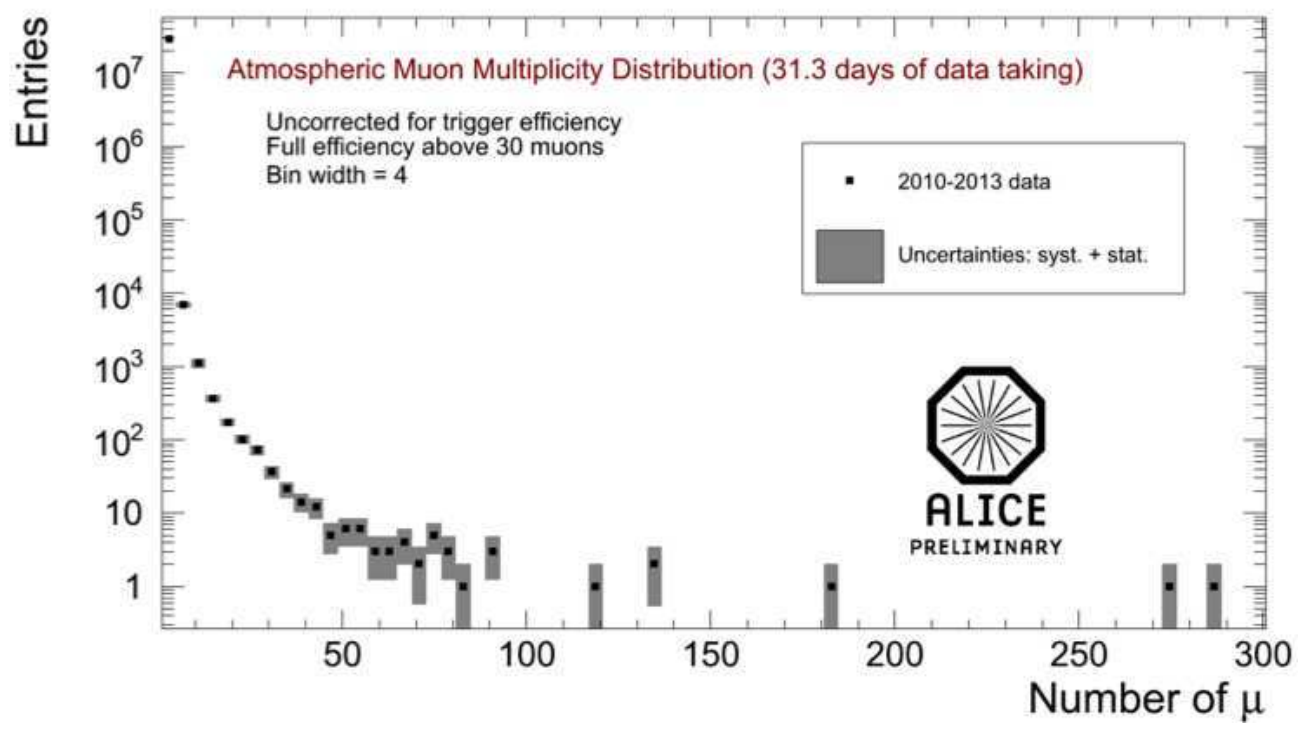

Figure 4: Atmospheric-muon multiplicity distribution (data collected by ALICE between 2010 and 2013)

There are 6 events with a number of muons greater than 100. As expected, a smooth distribution can be observed up to a muon multiplicity around 70. The MMD reconstructed by ALICE is very similar to that obtained by the LEP experiments [1,2], which could not be explained by Monte Carlo models.

\subsection{Study of MMD with Monte Carlo}

In this section, we are going to investigate the MMD, and in particular the rate and nature of high muon-multiplicity events (HMM, those events with a number of muons greater than 100).

Hadronic interactions are often described through phenomenological models implemented in Monte Carlo programs. To study the MMD, we have used CORSIKA [15] version 6990 and 7350 with QGSJET as interaction model $[12,13]$. The parameters used in the Monte Carlo simulations were the following:

- two independent samples were generated: pure $\mathbf{p}$ (lighter composition) and $\mathbf{F e}$ (extremely heavy composition),

- energy of the primary cosmic-ray: $10^{14}<10^{18} \mathrm{eV}$,

- usual power law spectrum $E^{-\gamma}$ with $\gamma=2.7$ for energies below the knee and $\gamma=3.0$ for energies above the knee, 
- the total (all-particle) absolute flux of the cosmic-rays was taken from [16],

- the core of each shower was scattered with a flat random distribution at surface level, in an area of $205 \times 205 \mathrm{~m}^{2}$ centered around the ALICE apparatus,

- the muons arriving at the surface level are propagated through the rock taking into account the geometry of the cavern where ALICE is located,

- the muons crossing the TPC are reconstructed with the same algorithm as the real data.

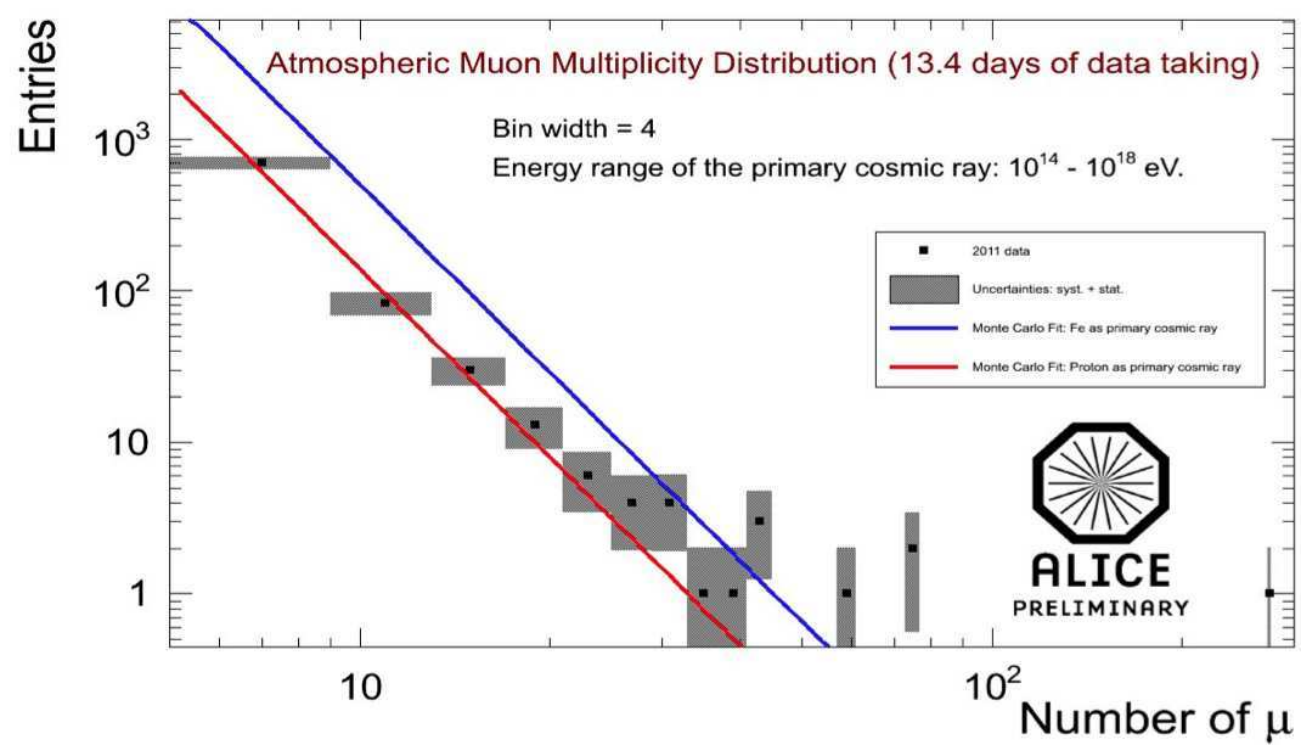

Figure 5: Atmospheric-muon multiplicity distribution of the data compared with the fit obtained with CORSIKA 6990 for proton (red line) and Fe (blue line)

The data points are, as expected, in between the pure proton composition (light elements) and pure Fe (heavy elements). The lower multiplicities (lower primary energies) are closer to pure proton (see Fig. 5).

Events with more than 100 atmospheric muons reconstructed by the ALICE-TPC are due to primary cosmic-rays with an energy larger than $10^{16} \mathrm{eV}$. To estimate the rate of the high muonmultiplicity events with the Monte Carlo models, a simulation of one year of effective data taking with Corsika 6990 (QGSJET II-03) and Corsika 7350 (QGSJET II-04, calibrated with TOTEM results on $\left.\sigma_{p p}^{t o t / e l}[17]\right)$ was done:

- energy of the primary cosmic-ray: $10^{16}<E<10^{18} \mathrm{eV}$,

- zenith angle range $0^{0}-50^{0}$

The fluctuations of these rare events are relatively small in the Monte Carlo (which corresponds to 1 year of data) but are quite large in the real data (31 days of data taking). Within the large uncertainties quoted in Table 1, there is agreement between the HMM rate observed in data and in the Monte Carlo calculation which makes use of CORSIKA supposing an heavy composition at these energies $\left(E>10^{16} \mathrm{eV}\right)$. 
Table 1: Comparison of the HMM event rate obtained with the full simulation and from measurement.

\begin{tabular}{l|cc|cc|c}
\hline \multirow{2}{*}{ HMM events } & \multicolumn{2}{|c|}{ CORSIKA 6990 } & \multicolumn{2}{c|}{ CORSIKA 7350 } & \\
& \multicolumn{2}{|c|}{ QGSJET II-03 } & \multicolumn{2}{|c|}{ QGSJET II-04 } & Data \\
& proton & iron & proton & iron & \\
\hline \hline Period [days per event] & 15.5 & 8.6 & 11.6 & 6.0 & 6.2 \\
Rate $\left[\times 10^{-6} \mathrm{~Hz}\right]$ & 0.8 & 1.3 & 1.0 & 1.9 & 1.9 \\
Uncertainty (\%) (syst + stat) & 13 & 16 & 8 & 20 & 49 \\
\hline
\end{tabular}

\section{Conclusions}

In the period 2010 to 2013, ALICE collected about 31 days of dedicated cosmic-ray data. The analysis of the collected data and its comparison with the Monte Carlo suggest a mixed composition with an increasing average mass of the primary cosmic ray at higher energies. The obtained MMD is in agreement with most experiments working in the energy range around the knee [1,2].

Using CORSIKA 6990/7350 (QGSJET II-03/04) as hadronic interaction model we are able to simulate these events and to reproduce, within relatively large uncertainties, their rate. It seems that most of the HMM are due to iron or heavy nuclei with an energy greater than $10^{16} \mathrm{eV}$ and a shower core located near ALICE.

Acknowledgment This work was supported by CONACyT México ALICE Network and Basic Science projects under grant numbers 260440 and 24408 (CB-2015-2018). The author also thanks to the VIEP-BUAP ALICE project.

\section{References}

[1] V. Avati et.al.Astropart. Phys. 19 (2003) 513-523.

[2] DELPHI Collaboration, .Nucl. Phys. B Proc. Suppl. 138 295-298 (2005).

[3] K. Aamodt et al., (ALICE Collaboration), JINST 3 (2008), S08002

[4] Pierre Auger Collaboration, Nuclear Instr. and Methods in Physics Research A 523 50-95 (2004)

[5] M. Aglietta et al., (EAS-TOP Collaboration), Astropart. Phys. 10 1-9 (1999)

[6] M.A.K. Glasmacher et al., (CASA-MIA Collaboration), Astropart. Phys. 10 291-302 (1999)

[7] T. Antoni et al., (KASKADE Collaboration), Astropart. Phys. 14245 (2001)

[8] G. Battistoni et. al. (NUSEX Collaboration), II Nuovo Cimento Vol. 9C, N. 2, 196-209 (1986)

[9] M. Ambrosio et. al., (MACRO Collaboration), Phys. Rev. D52 3793 (1995) .

[10] P. Kuusiniemi et. al., (EMMA Collaboration), Journal of Physics: Conference Series 409 012067 (2013). 
[11] J. Alme et al., Nuclear Instr. and Methods in Physics Research A 622 316-367 (2010)

[12] Ostapchenko, S., Nucl. Phys. Proc. Suppl. 151 143-146 (2006)

[13] Ostapchenko, S., Phys. Rev. D 74014026 (2006)

[14] B. Alessandro et al., (ALICE Collaboration), J. Phys, G: Nucl. Part. Phys. 321295 (2006).

[15] D. Heck et al., Report FZKA 6019 (1998), Forschungszentrum Karlsruhe, https : / /www . ikp.kit.edu/corsika/.

[16] J. R. Horandel, Astrop. Phys. 19 193-220 (2003) .

[17] G. Antchev et al. (TOTEM Collaboration), Euro-phys. Lett.96, 21002 (2011)

[18] ALICE Collaboration, http://arxiv.org/abs/1507.07577 